\title{
Real-Time Nanoplasmonic Sensor for IgG Monitoring in Bioproduction
}

\author{
Thuy Tran ${ }^{1}$, Olof Eskilson ${ }^{1}$, Florian Mayer ${ }^{2}{ }^{(0)}$, Robert Gustavsson ${ }^{2}$, Robert Selegård ${ }^{1}$ (), \\ Ingemar Lundström ${ }^{3}$, Carl-Fredrik Mandenius ${ }^{2}$, Erik Martinsson ${ }^{4}$ and Daniel Aili 1,*(D) \\ 1 Laboratory of Molecular Materials, Division of Biophysics and Bioengineering, Department of Physics, \\ Chemistry and Biology, Linköping University, 58183 Linköping, Sweden; thuy.tran@liu.se (T.T.); \\ olof.eskilsson@liu.se (O.E.); robert.selegard@liu.se (R.S.) \\ 2 Laboratory of Biotechnology, Division of Biophysics and Bioengineering, Department of Physics, \\ Chemistry and Biology, Linköping University, 58183 Linköping, Sweden; florian.mayer1994@gmx.at (F.M.); \\ robert.gustavsson@liu.se (R.G.); carl-fredrik.mandenius@liu.se (C.-F.M.) \\ 3 Sensor and Actuator Systems, Department of Physics, Chemistry and Biology, Linköping University, \\ 58183 Linköping, Sweden; ingemar.lundstrom@liu.se \\ 4 ArgusEye AB, Spannmålsgatan 55, 58336 Linköping, Sweden; erik.martinsson@arguseye.se \\ * Correspondence: daniel.aili@liu.se
}

Received: 21 September 2020; Accepted: 14 October 2020; Published: 16 October 2020

check for updates

\begin{abstract}
Real-time monitoring of product titers during process development and production of biotherapeutics facilitate implementation of quality-by-design principles and enable rapid bioprocess decision and optimization of the production process. Conventional analytical methods are generally performed offline/at-line and, therefore, are not capable of generating real-time data. In this study, a novel fiber optical nanoplasmonic sensor technology was explored for rapid IgG titer measurements. The sensor combines localized surface plasmon resonance transduction and robust single use Protein A-modified sensor chips, housed in a flexible flow cell, for specific IgG detection. The sensor requires small sample volumes $(1-150 \mu \mathrm{L})$ and shows a reproducibility and sensitivity comparable to Protein $\mathrm{G}$ high performance liquid chromatography-ultraviolet (HPLC-UV). The dynamic range of the sensor system can be tuned by varying the sample volume, which enables quantification of IgG samples ranging from 0.0015 to $10 \mathrm{mg} / \mathrm{mL}$, without need for sample dilution. The sensor shows limited interference from the sample matrix and negligible unspecific protein binding. IgG titers can be rapidly determined in samples from filtered unpurified Chinese hamster ovary $(\mathrm{CHO})$ cell cultures and show good correlation with enzyme-linked immunosorbent assay (ELISA).
\end{abstract}

Keywords: PAT; IgG titer; real-time; on-line; bioprocess; nanoplasmonic

\section{Introduction}

Efficient and cost-effective production of biologics requires the ability to accurately monitor and control the production process. Guidelines concerning process analytical technologies (PATs) released by the U.S. Food and Drug Administration (FDA) in 2004 [1] initiated a large interest in development and implementation of technologies for near real-time or real-time monitoring of key performance indicators (KPIs) and critical quality attributes (CQAs) in bioproduction [2-4].

Therapeutic antibodies are currently the largest group of biopharmaceuticals. Until 2019, a total of 79 therapeutic immunoglobulin G (IgG) monoclonal antibodies (mAbs) had been approved by the U.S. FDA $[5,6]$. Techniques for process development and production of mAbs using mammalian cell cultures have been evolving rapidly, resulting in product titers ranging from about 1 to $10 \mathrm{mg} / \mathrm{mL}$ using $\mathrm{CHO}$ cell cultures [7-10]. Possibilities to monitor IgG titers are central for optimizing process conditions and 
to achieve high yields as well as consistent and high product quality. The IgG titer is thus naturally an important KPI in production of mAbs. There are several techniques that can be used for measuring IgG titers, such as enzyme-linked immunosorbent assay (ELISA) [11], protein A/G high performance liquid chromatography-ultraviolet (Protein A/G-HPLC-UV) [12,13], surface plasmon resonance (SPR) [14,15], biolayer interferometry (BLI) [16] and capillary electrophoresis-mass spectrometry (CE-MS) [17]. Among these, protein A/G-HPLC-UV has been the most widely used method for bioprocess monitoring and validation due to its high selectivity, robustness and reliable results. However, these methods typically require multiple sample preparation steps and are difficult to automize and implement as on-line/in-line techniques and are, therefore, generally performed at-line or off-line. Possibilities to acquire specific near real-time or real-time data of IgG titers are thus limited. With the increasing demand for applying PAT tools in bioprocessing, efforts have been made to reduce the turn-around time of available analytical methods. Pedersen et al. introduced a method using flow-induced dispersion analysis that could quantify IgG in cell culture broth in less than $15 \mathrm{~min}$ [18]. Swartz et al. developed a quick and cost-effective antibody-nanocage turbidity assay for rapid measurements of IgG in cell culture samples [19]. These methodologies were, however, not possible to integrate for on-line measurements. An on-line/at-line system using a portable ultraperformance liquid chromatography (UPLC) system interfaced with an UPLC-process sample manager was recently developed by Letha et al., indicating the potential for more rapid IgG titer measurements [20]. The technique, however, requires complex equipment and sample handling, including purification, which complicates implementation in bioproduction. Despite large efforts to improve the speed of IgG quantification methods, there is still a lack of robust cost-effective sensor systems that allow for specific on-line real-time titer measurements.

Localized surface plasmon resonance (LSPR)-based sensors, or nanoplasmonic sensors, have emerged as versatile techniques for label-free biomolecular interaction analysis [21-23]. LSPR is an optical phenomenon caused by coherent electron oscillations in metal nanostructures. The resonance conditions are sensitive to changes in refractive index in the immediate vicinity of the nanostructures, which enable detection of analyte binding to immobilized ligands. In comparison to benchtop surface plasmon resonance (SPR) sensor systems, LSPR sensors require significantly less complex optical setups and are less sensitive to temperature fluctuations and changes in the sample background. However, SPR can enable label-free at-line and off-line monitoring of IgG titers in samples from upstream process steps but, due to the narrow and low dynamic range, with reported values spanning from 13-30 $\mu \mathrm{g} / \mathrm{mL}$ [24], 0.019-9.6 $\mu \mathrm{g} / \mathrm{mL}$ [15] to 2-200 $\mu \mathrm{g} / \mathrm{mL}$ [14], extensive sample dilution is required.

Here, we explore a novel LSPR-based sensor technology that combines nanoplasmonic sensing with fiber optics that is possible to integrate for specific on-line/in-line biodetection in real-time. The sensor technology, schematically described in Figure 1, was developed in close collaboration with ArgusEye AB (Linköping, Sweden) with the purpose of enabling more efficient bioprocess monitoring. To our knowledge this is the first LSPR-based sensor system designed for on-line/in-line bioprocess monitoring. The analytical performance of the sensor system for detection of IgG in complex samples was evaluated using samples from both upstream and downstream process steps, and data was in good agreement with results from ELISA and protein G-HPLC-UV. The sensor has a short response time (seconds to minutes) and a large and tunable dynamic range (from $0.0015 \mathrm{mg} / \mathrm{mL}$ to about $30 \mathrm{mg} / \mathrm{mL}$ ) and was capable of rapid IgG quantification in cell-free samples directly without any need for sample pretreatment. Successful integration of this novel sensor technology in bioproduction and process development could improve process understanding, facilitate implementation of quality by design $(\mathrm{QbD})$ and development of strategies for continuous processing. 
A

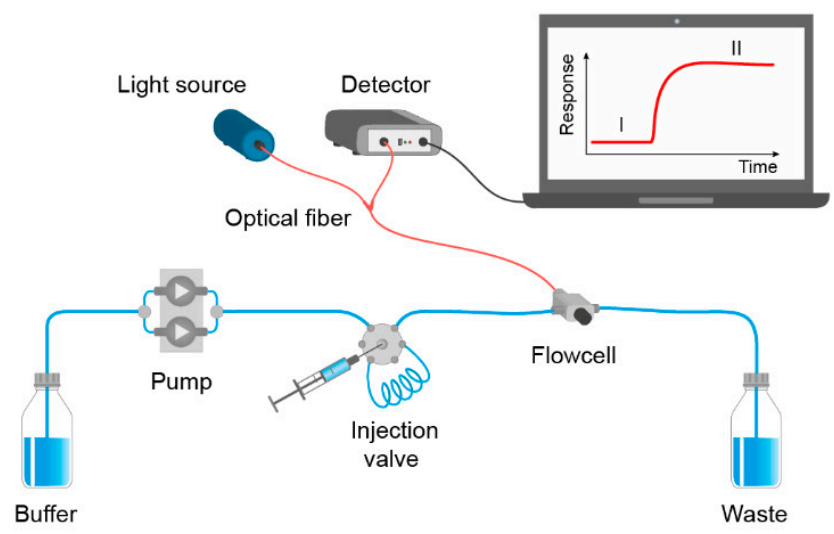

B

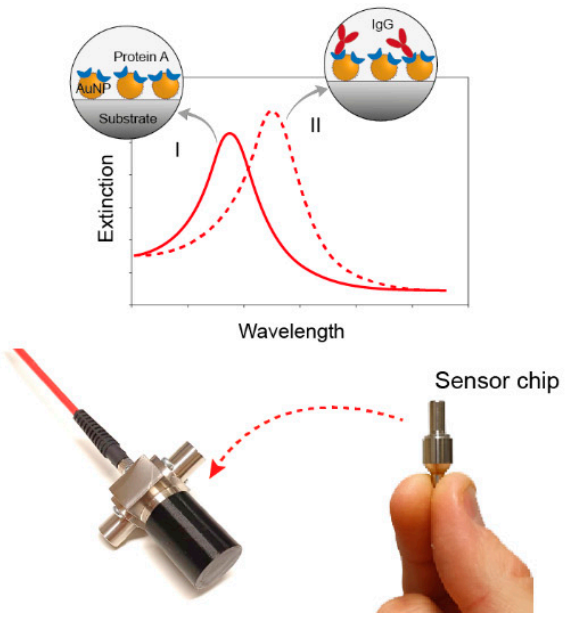

Figure 1. (A) Schematic illustration of the sensor system setup. Samples with IgG were injected into the flow cell containing the sensor chip. (B) Nanoplasmonic sensor chips were functionalized with Protein A for specific recognition of IgG. Binding of IgG to the sensor chip results in a concentration-dependent redshift of the LSPR peak maximum. The sensor signal is transmitted to the detector using fiber optics and was monitored in real-time using a dedicated software.

\section{Materials and Methods}

\subsection{Chemicals and Reagents}

$N$-Ethyl- $N^{\prime}$-(3-dimethylaminopropyl)carbodiimide (EDC), N-Hydroxysuccinimide (NHS), Bovine serum albumin (BSA), ethanolamine, 4-Morpholineethanesulfonic acid (MES), purified IgG from human serum $(5.5 \mathrm{~g} / \mathrm{L})$ were obtained from Sigma-Aldrich (St. Louis, MO, USA). Protein A was supplied by Medicago AB (Upsala, Sweden). Hyclone ActiPro Medium, Hyclone Cell boost 7a, Hyclone Cell boost $7 \mathrm{~b}$ were purchased from GE Healthcare Life Sciences (Logan, UT, USA). Sheep antihuman IgG gamma chain and sheep antihuman IgG gamma chain peroxidase conjugate were purchased from by The Binding Site Group Ltd (Birmingham, UK). TMB (Tetramethylbenzidine) substrate kit, Novex sharp prestained protein standard, Bolt LDS sample buffer, Bolt 4-12\% Bis-Tris Plus gels, MOPS (4-Morpholinepropanesulfonic acid) SDS running buffer, SimplyBlue SafeStain solution were purchased from Thermo Fisher Scientific (Rockford, IL, USA). GlutaMAX ${ }^{\mathrm{TM}}(100 \times)$ was from Life Technologies/Gibco (Carlsbad, CA, USA).

\subsection{Ligand Immobilization}

Ligand immobilization to carboxyl sensor chips (ArgusEye AB, Linköping, Sweden) was carried out using carbodiimide (EDC/NHS) coupling chemistry. A ( $v / v 1: 1)$ mixture of $20 \mu \mathrm{L}$ of $0.4 \mathrm{M}$ EDC and $0.1 \mathrm{M}$ NHS was added to the sensor surfaces, and carboxyl groups were activated for $45 \mathrm{~min}$. After rinsing with Milli-Q water $\left(18.2 \mathrm{M} \Omega \mathrm{cm}^{-1}\right), 20 \mu \mathrm{L}$ of $0.5 \mathrm{mg} / \mathrm{mL}$ ligand solution was added and the coupling reaction was carried out for $2 \mathrm{~h}$. Unreacted carboxyl groups were deactivated by using of $20 \mu \mathrm{L}$ of $1 \mathrm{M}$ ethanolamine ( $\mathrm{pH}$ 8.5) for $30 \mathrm{~min}$. The sensor substrates were rinsed and stored in PBS buffer before being inserted into the LSPR system.

\subsection{LSPR Measurements}

The fiber optical sensor system was provided by ArgusEye AB and includes a flow cell and an optical detection unit comprising a halogen light source and a spectrophotometer. Sensor chips functionalized with Protein A (ProtA) or BSA were inserted into the flow cell and equilibrated with PBS buffer at a flow rate of $1 \mathrm{~mL} / \mathrm{min}$ using an HPLC pump for about $15 \mathrm{~min}$ to ensure stable baseline. Samples were manually injected into the flow cell and the sensor signal was continuously recorded using software developed by ArgusEye. Purified monomeric IgG and filtrated bioreactor samples (provided 
by BioInvent International AB, Lund, Sweden) were used to validate the analytical performance of the sensor system. The system was run in a normal laboratory setting at room temperature without any additional temperature control.

\subsection{CHO Cell Cultivation}

A recombinant $\mathrm{CHO}$ cell line (CHOK-1 derivate cell line) producing human IgG was provided from Cobra Biologics Ltd. (Newcastle, UK). The $\mathrm{CHO}$ cell line was cultured in spinner flasks in fed-batch mode at $37{ }^{\circ} \mathrm{C}$ in a humidified atmosphere containing $7.5 \% \mathrm{CO}_{2}$. HyClone Actipro ${ }^{\mathrm{TM}}$ medium supplemented with 3\% GlutaMAX ${ }^{\mathrm{TM}}(100 \times)$ was used as culture media. At day three of culture, a daily feed of $2 \%$ HyClone Cell Boost ${ }^{\mathrm{TM}} 7 \mathrm{a}$ and $0.2 \%$ HyClone Cell Boost $^{\mathrm{TM}} 7 \mathrm{~b}$ of the culture volume was applied. Samples for analysis were withdrawn from the culture daily, centrifuged $(200 \times g$, $5 \mathrm{~min}$ ) and the supernatant was $0.2 \mu \mathrm{m}$ filtered before storage at $-20{ }^{\circ} \mathrm{C}$.

\subsection{ELISA IgG Quantification}

ELISA assays were performed using a general colorimetric sandwich ELISA procedure. Briefly, Sheep antihuman IgG gamma chain was coated on a Maxisorp Nunc-Immuno well plate (Thermo Fisher Scientific) and incubated overnight at $4{ }^{\circ} \mathrm{C}$. After rinsing, standards (Purified IgG from human serum) and samples were added and incubated for $2 \mathrm{~h}$ at $37^{\circ} \mathrm{C}$. Samples were discarded followed by adding sheep antihuman IgG gamma chain peroxidase conjugate and incubated for $1 \mathrm{~h}$ at $37^{\circ} \mathrm{C}$. Color development using TMB solution was done at room temperature and the reaction was stopped using $\mathrm{H}_{2} \mathrm{SO}_{4}$. Absorbances at $450 \mathrm{~nm}$ and $620 \mathrm{~nm}$ were measured for quantification.

\subsection{SDS-PAGE IgG Quantification}

Novex sharp prestained protein standards were used as molecular weight standards. Samples and standards (Purified IgG from human serum) were mixed with Bolt LDS sample buffer and purified water and incubated for $10 \mathrm{~min}$ at $70{ }^{\circ} \mathrm{C}$. The samples were loaded on Bolt $4-12 \%$ Bis-Tris Plus gels, and electrophoresis was performed at $200 \mathrm{~V}$ for between 35 to 45 min using an XCell SureLock electrophoresis cell (Thermo Fisher Scientific, Carlsbad, CA, USA) and a E443 power supply (Consort). Bolt MOPS SDS running buffer was used as running buffer. Gels were stained by using SimplyBlue SafeStain solution with $1 \mathrm{~h}$ incubation and destained using purified water for $1 \mathrm{~h}$. The gels were scanned for further evaluation and quantification.

\section{Results and Discussion}

\subsection{LSPR Detection Setup}

The LSPR measurements were conducted using a novel fiber optical sensor system comprised of a flow cell designed for real-time on-line and in-line bioproduction monitoring connected to a halogen light source and a detector using fiber optics (Figure 1). The flow cell was produced in medical grade stainless steel to tolerate both cleaning in place and high pressures. Single use sensor chips, modified with gold nanostructures and functionalized with Protein A, were docked into the flow cell to enable specific IgG detection. Analyte binding results in a change in refractive index detected as a shift in the LSPR resonance conditions. The sensor response (in picometer, pm) was recorded in real time and the sensorgrams indicated both analyte concentration and binding kinetics. To simulate on-line conditions, the flow cell was connected to a manual injection valve. An HPLC pump was used to control the flow, and samples were injected into the flow cell using an injection valve with replaceable sample loops with different volumes. In a real on-line detection setup, this would be replaced by an automated system where sample fractions are collected using software-controlled valves. 


\subsection{Protein A Immobilization}

The sensor chips were fabricated in medical grade stainless steel modified with nanoplasmonic gold nanostructures and further functionalized with a robust surface chemistry that enables covalent immobilization of ligands using carbodiimide chemistry. The $\mathrm{pH}$ of the coupling buffer influences both the net charge of ProtA ( $\mathrm{pI}$ (isoeletric point) = 5.1) and the net-charge of the sensor surface, which can affect the electrostatic attraction between the sensor surface and the protein during the coupling reaction. ProtA with a concentration of $0.5 \mathrm{mg} / \mathrm{mL}$ prepared in three different buffers, acetate $\mathrm{pH} 4.0$, MES pH 6.0, and phosphate buffer $\mathrm{pH} 7.0$ was used to compare the influence of $\mathrm{pH}$ on coupling efficiency (Appendix A, Figure A1). The acidic buffers were found to give only marginally higher IgG binding responses compared to neutral $\mathrm{pH}$, which indicated that the effect of $\mathrm{pH}$ in this case was not significant. ProtA immobilization was, therefore, conducted using $10 \mathrm{mM} \mathrm{MES} \mathrm{pH} 6.0$ in all further experiments.

\subsection{Sensor Evaluation}

\subsubsection{Unspecific Binding}

The binding of IgG to the ProtA sensor chips was investigated using a simulated on-line setup where samples were manually injected into the sensor flow system using an HPLC injection port. Injection of IgG $(1 \mathrm{mg} / \mathrm{mL})$ resulted in an LSPR response of about $2000 \mathrm{pm}$ (Figure 2A,B). In contrast, when using bovine serum albumin (BSA) as a ligand instead of ProtA, no significant binding could be detected (Figure 2A,B). The contribution from unspecific binding of IgG to the sensor signal was consequently below the detection limit of the sensor. When injecting undiluted cell culture media $(1 \mathrm{~mL})$ to a ProtA chip, a negative response was obtained, followed by a very small increase in the baseline, corresponding to only about $2 \%$ of the specific IgG binding. The negative shift is a consequence of the yellow color of the cell culture medium that interferes with the baseline. After the injection has passed through the flow cell, the baseline stabilizes and returns to normal. Consequently, the readout of protein binding to sensor surface must, in this case, be assessed after the injection. Here, the responses during the dissociation phase $(\mathrm{t}>150 \mathrm{~s}, 40.6 \mathrm{pm})$ better reflect the binding and indicate a negligible unspecific interaction between components in the cell culture medium and the ProtA sensor chip (Figure 2B). The negative dip can also, if needed, be subtracted by including a reference cell to the system. In these experiments, the sample volume was $1 \mathrm{~mL}$ and was injected into the system at a flow rate of $1 \mathrm{~mL} / \mathrm{min}$. With smaller sample volumes, the influence on sample color can be eliminated and effects of unspecific binding further reduced (vide infra).
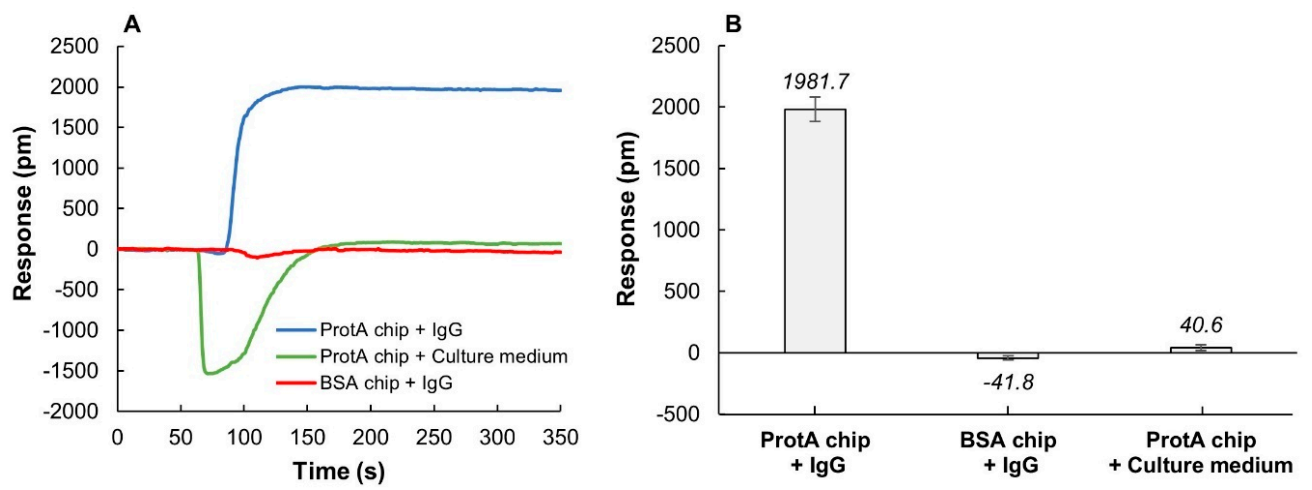

Figure 2. Unspecific binding evaluation using bovine serum albumin (BSA) chips and cell culture

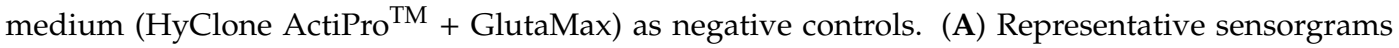
showing the binding of IgG $(1 \mathrm{mg} / \mathrm{mL})$ to a ProtA chip and a BSA chip, and the binding of cell culture medium to a ProtA chip. (B) Comparison of specific and unspecific binding responses. Relative binding responses at $\mathrm{t}=250 \mathrm{~s}$ were averaged ( $\mathrm{n}=3$ sensor chips), and error bars show standard deviations. Injection volume was $1 \mathrm{~mL}$. 


\subsubsection{Regeneration Scouting}

As in most other affinity-based biosensors, the ProtA sensor chips can be regenerated to allow for multiple consecutive measurements. To evaluate the efficiency of the regeneration process, different concentrations of $\mathrm{IgG}$ were injected followed by a short $(1 \mathrm{~min}) 10 \mathrm{mM}$ glycine buffer $\mathrm{pH} 2.5$ pulse (Figure 3A). The regeneration buffer efficiently disrupted the interaction between IgG and ProtA and the signal returned to baseline after a single regeneration pulse for the two lower $(0.0625$ and $0.156 \mathrm{mg} / \mathrm{mL}$ ) $\mathrm{IgG}$ concentrations. After a second regeneration pulse, the chip exposed to $1 \mathrm{mg} / \mathrm{mL} \mathrm{IgG}$ also returned close to baseline (Figure $3 \mathrm{~A}, \mathrm{~B}$ ). ProtA is fairly stable under these conditions but to verify the effect of the regeneration buffer on the IgG binding, eight cycles of IgG injections, each followed by two regeneration pulses, were conducted (Figure $3 \mathrm{C}$ ). Absolute and relative binding responses at the last injection were reduced by only 1.5 and $4.8 \%$, respectively, as compared to the first injection (Figure 3D). The ProtA chips are, consequently, both robust enough to allow for multiple consecutive measurements while efficiently preventing unspecific protein binding, which are important properties for PAT applications.
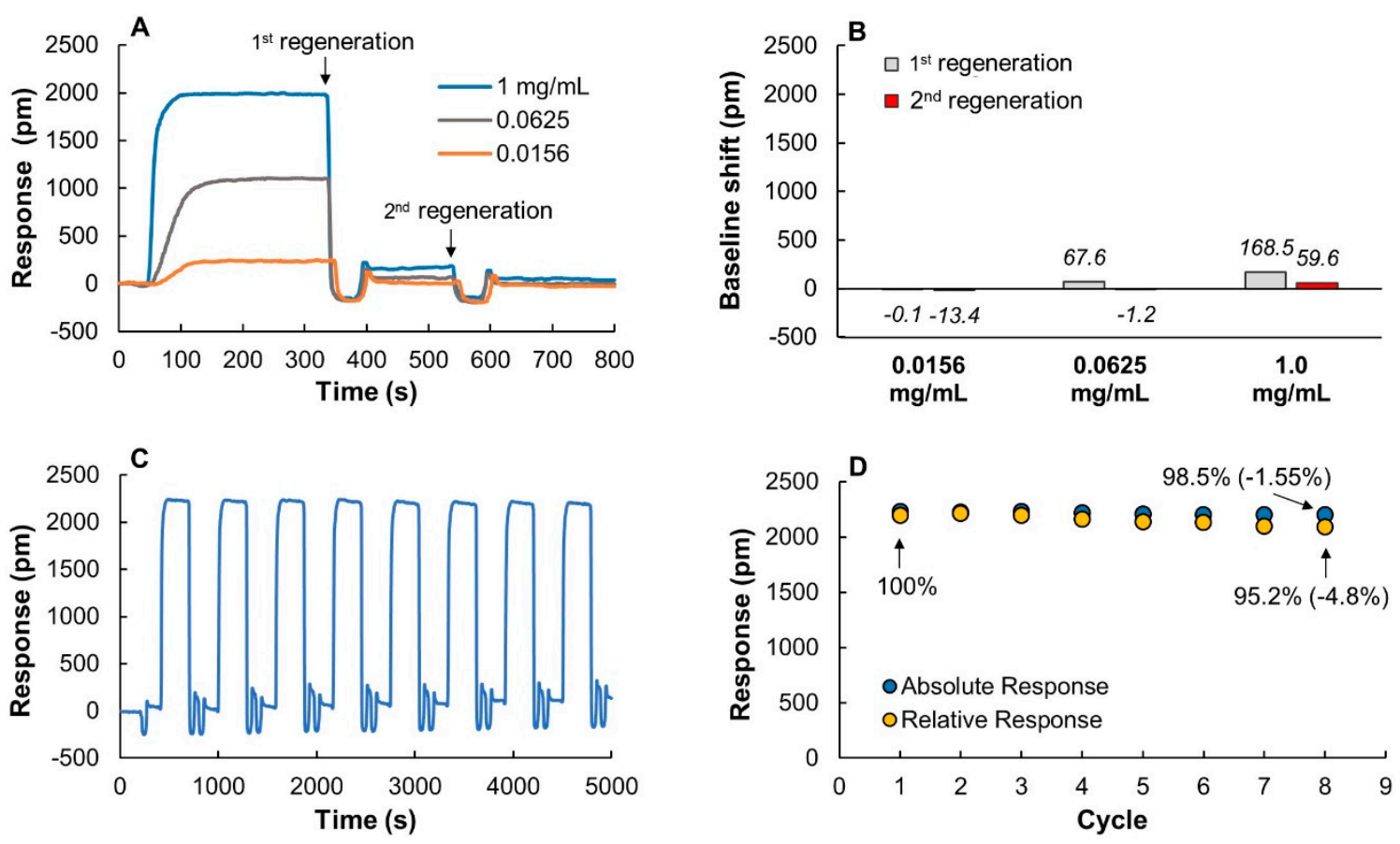

Figure 3. (A) Binding and regeneration profiles of IgG to a ProtA chip using three IgG concentrations: 0.0156, 0.0625 and $1 \mathrm{mg} / \mathrm{mL}$. (B) Baseline shift after first and second regeneration pulse. (C) Sensorgrams obtained after eight cycles of IgG injections $(1 \mathrm{mg} / \mathrm{mL})$ each followed by two regeneration pulses using $10 \mathrm{mM}$ glycine buffer $\mathrm{pH}$ 2.5. (D) Change in absolute and relative binding responses after each binding and regeneration cycle. The relative binding response is the difference between the absolute responses and the baseline before each IgG injection. The sample volume was $1 \mathrm{~mL}$ in all experiments.

\subsubsection{IgG Binding Reproducibility}

To test the reproducibility and robustness of the sensor system, binding responses from three different sensor chips were compared using IgG samples with different concentrations: $0.0156,0.0625$ and $2 \mathrm{mg} / \mathrm{mL}$ (Figure 4). The colored area in the sensorgrams indicate the 95\% confidence intervals for three independent sensor chips and two injection replicates per chip. Binding responses at $t=250 \mathrm{~s}$ were used to evaluate the variation between sensor chips and within sensor chips. Coefficient of variation $(\mathrm{CV})$ of the binding responses across sensor chips varied from 9.5 to $12.7 \%$, while with the same chip, the variations were only about 3 to $4 \%$ (Table 1 ). These results show that the data generated using the sensor system were highly reproducible, also when using different sensor chips. 


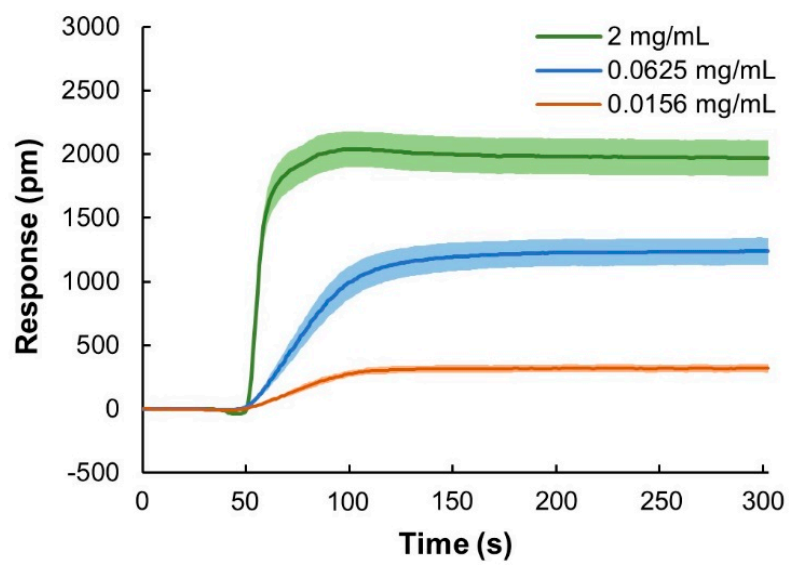

Figure 4. Evaluation of reproducibility of sensor chips. Mean responses from six runs using $n=3$ different chips and two injection replicates per chip using three different IgG concentrations, 0.0156, 0.0625 and $2 \mathrm{mg} / \mathrm{mL}$. The data show 95\% confidence intervals. Injection volume was $1 \mathrm{~mL}$.

Table 1. Coefficient of variation $(\mathrm{CV})$ of binding responses at $\mathrm{t}=250 \mathrm{~s}$ of three IgG concentrations: $0.015,0.0625$ and $2 \mathrm{mg} / \mathrm{mL}$. Between-chip CV was calculated based on the mean responses obtained from three different chips. Within-chip CV was the mean of CV calculated based on binding responses obtained from the same chip.

\begin{tabular}{cccc}
\hline Concentration (mg/mL) & $\mathbf{0 . 0 1 5 6}$ & $\mathbf{0 . 0 6 2 5}$ & $\mathbf{2}$ \\
\hline Between-Chip CV (\%) & 12.7 & 11.4 & 9.5 \\
Within-Chip CV (\%) & 2.6 & 3.3 & 4.3 \\
\hline
\end{tabular}

\subsubsection{Dynamic Range}

The ProtA sensor chips have a certain number of accessible IgG binding sites that depend on the surface concentration of immobilized ProtA and their relative orientation on the sensor surface and, therefore, show an upper limit in binding capacity. When fully saturated, the sensor response was about $2000 \mathrm{pm}$. The IgG concentration required to saturate the surface was found to depend on injection volume (Appendix A, Figure A2), which enables flexible tuning of the dynamic range. The dynamic range of the sensor system was investigated using three different injection volumes, $1 \mathrm{~mL}$, $20 \mu \mathrm{L}$ and $1 \mu \mathrm{L}$. The sensor surface was saturated at lower concentrations when increasing the injection volumes (Figure 5). By varying the injection volume in the range of $1 \mu \mathrm{L}$ to $1 \mathrm{~mL}$, the total dynamic range could be expanded to more than three orders of magnitude, from 0.0015 to about $30 \mathrm{mg} / \mathrm{mL}$. The linear range for quantification was estimated to be $0.0015-0.06,0.125-1.5$, and $0.5-10 \mathrm{mg} / \mathrm{mL}$ at $1 \mathrm{~mL}, 20 \mu \mathrm{L}$ and $1 \mu \mathrm{L}$ injection volumes, respectively. Compared to ELISA [25,26], which is normally performed at the $\mu \mathrm{g} / \mathrm{mL}$ level, and to a recently developed protein A-HPLC-UV method [27] with an improved linear range of $0.01-5.2 \mathrm{mg} / \mathrm{mL}$, the sensor system used here showed a substantially higher upper limit for quantification. The linear range reported in present work covers the range of normal cell culture titers $(5-10 \mathrm{mg} / \mathrm{mL})$ that can be routinely achieved in industrial cell culture processes nowadays $[7,8]$. Therefore, bioreactor samples can be analyzed without the need of dilution, which simplifies sample handling and facilitates on-line/in-line detection.

\subsubsection{IgG Quantification in Bioreactor Samples}

Filtrated crude bioreactor samples, provided from an industrial bioproduction process with a known concentration of $2.3 \mathrm{mg} / \mathrm{mL}$ determined by Protein G-HPLC-UV, were used to validate the method for quantification. Nine $\operatorname{IgG}$ standards diluted from a monomeric sample of the same $\operatorname{IgG}$ were used to calibrate the system, and two injections of sample were used for concentration determination. A full recording of both standards and samples are shown in Figure 6A. Extracted sensorgrams of IgG 
concentrations, $0.5,1,2,3,5$, and $10 \mathrm{mg} / \mathrm{mL}$ defining the linear range are shown in Figure 6B. Interestingly, although the bioreactor samples had a distinct yellow color, the negative dip seen during the association phase when using $1 \mathrm{~mL}$ injection volumes (Figure 2A) was not observed here when using a smaller sample volume of $1 \mu \mathrm{L}$. The linearity of the standard curve was high with $R^{2}=0.9962$, and the determined concentration of $2.5 \mathrm{mg} / \mathrm{mL}$ was in very good agreement with the expected value (Figure 6C).

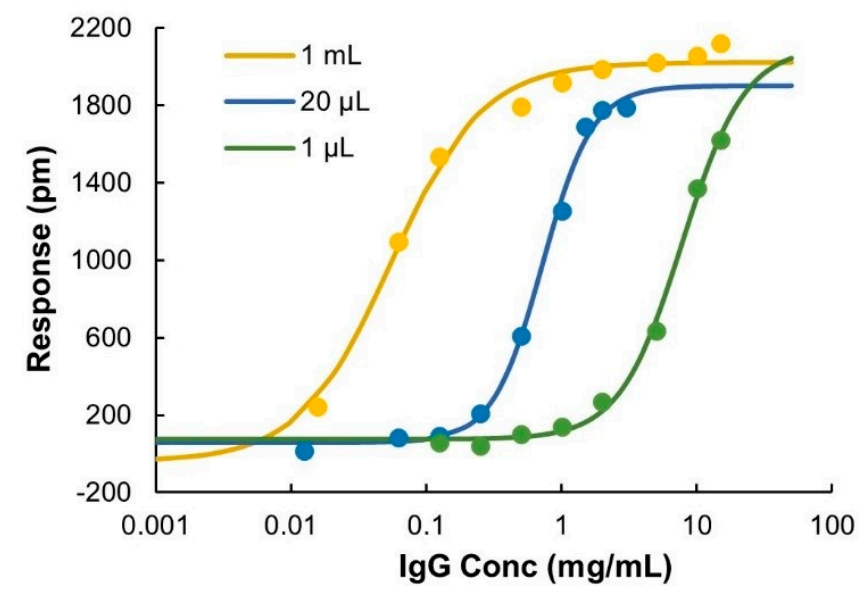

Figure 5. Binding responses at $\mathrm{t}=250 \mathrm{~s}$ versus $\mathrm{IgG}$ concentrations fitted to sigmoidal curves when using various sample injection volumes, $1 \mu \mathrm{L}, 20 \mu \mathrm{L}$, and $1 \mathrm{~mL}$.
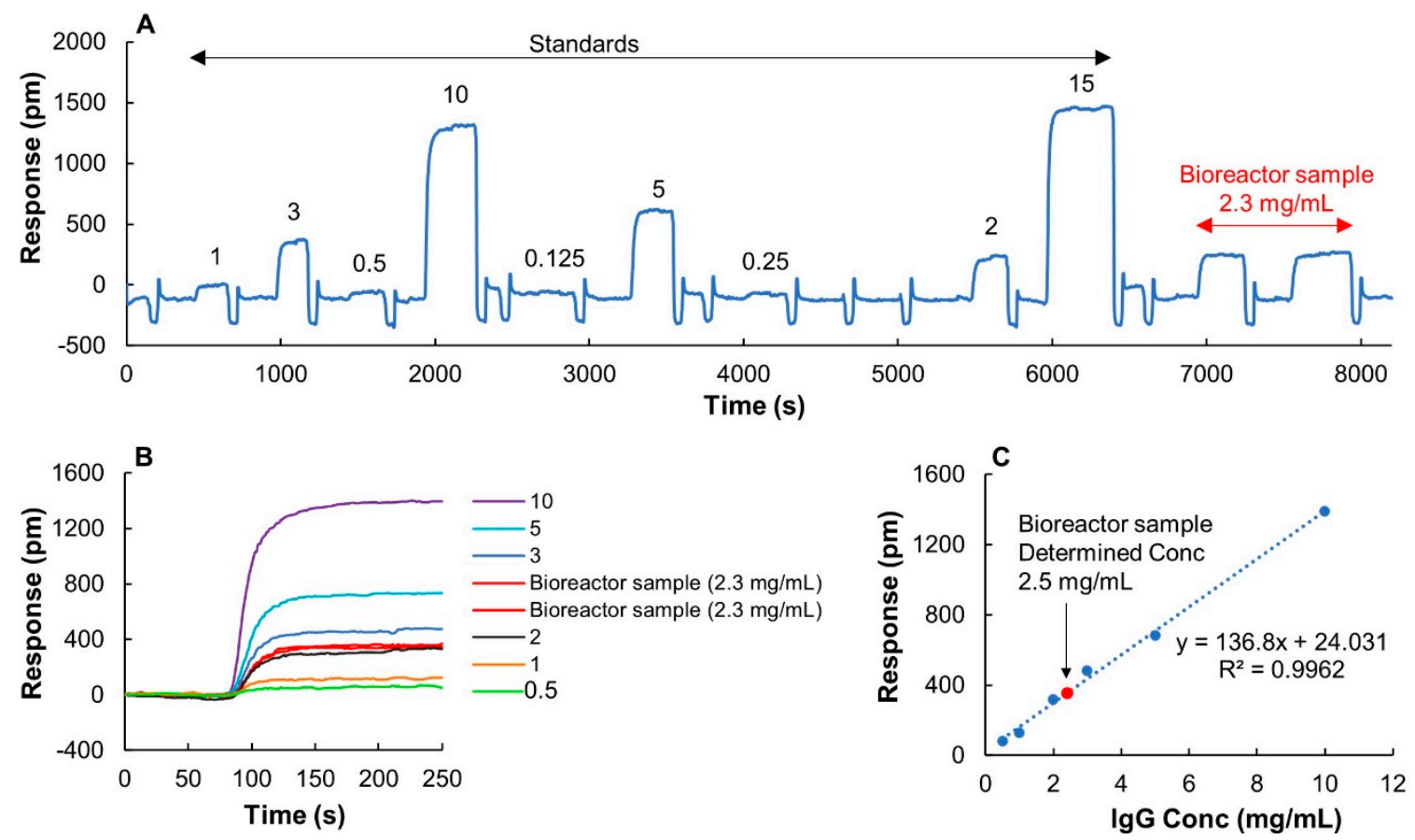

Figure 6. Quantification of IgG in filtrated upstream samples from a bioreactor cell culture. The sample has an IgG concentration of $2.3 \mathrm{mg} / \mathrm{mL}$ determined by traditional Protein G-HPLC-UV. (A) A full run of nine IgG standards with various concentrations starting from 0.125 to $15 \mathrm{mg} / \mathrm{mL}$ and two injections of the bioreactor sample. $1 \mu \mathrm{L}$ of samples was used for measurements. Concentrations are displayed on top of each individual sensorgram. The sensor chip was regenerated using $10 \mathrm{mM}$ glycine buffer $\mathrm{pH}$ 2.5 after each sample injection. (B) Extracted and normalized sensorgrams of the sample and six IgG standards $(0.5,1,2,3,5,10 \mathrm{mg} / \mathrm{mL})$ used for making the calibration curve. (C) Calibration curve and determined concentration of sample based on two replicates. 


\subsection{Measurement of IgG Titers during Cell Culture}

The possibility to rapidly and accurately determine IgG titers over a wide concentration range in an upstream process step was further assessed using a CHO fed-batch culture. Samples collected daily for 15 days from a $\mathrm{CHO}$ cell culture were measured using the same sensor chip and IgG titers were determined. A full run, including standards and samples, is shown in Figure 7A. Negative dips in the sensorgrams of samples (D0-D14) were caused by the color of the media but did not interfere with the analysis of the data. Based on the linear range (Figure 7B) and expected concentrations of IgG, a sample volume of $150 \mu \mathrm{L}$ was used for all the measurements. The binding responses leveled off at day 11 (Figure 7C), indicating that a maximum titer was achieved. Since the binding responses of samples at day 11,12, 13 and 14 were lower than the maximum binding capacity (2036 $\pm 120 \mathrm{pm})$, and were within the linear range of the sensor chip, the sensor surface was never saturated.
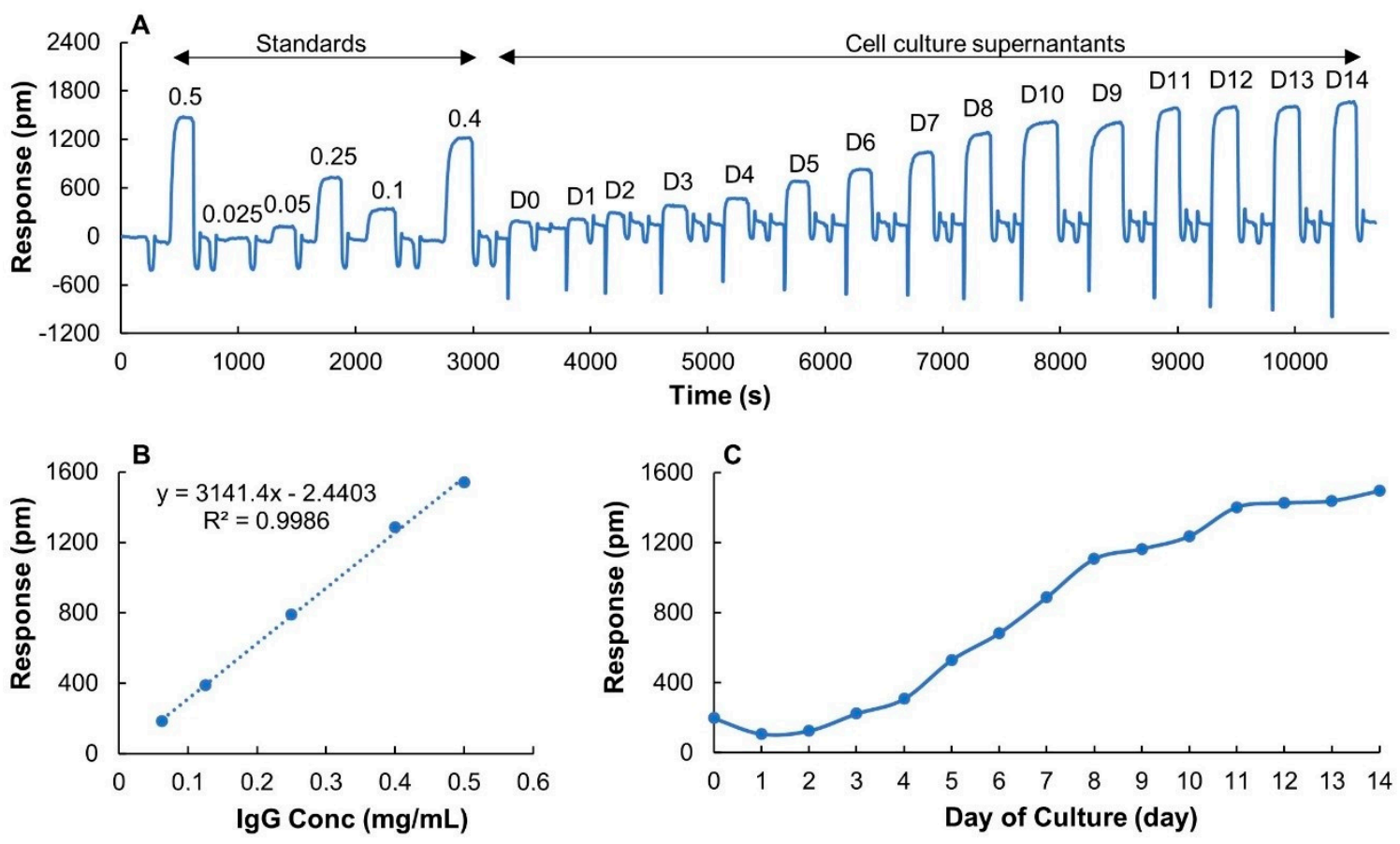

Figure 7. LSPR measurement of filtrated cell culture samples collected daily over two weeks of cultivation. (A) A full run of six IgG standards (diluted from downstream monomeric IgG sample $15 \mathrm{mg} / \mathrm{mL}$ ) with different concentrations marked on top of each sensorgram and 15 cell culture samples collected from day 0 to day 14 (D0-D14). Injection volume was $150 \mu \mathrm{L}$. (B) Calibration curve. (C) Binding responses of samples over cultivation time.

In Figure 8A, the LSPR data are compared to results from ELISA and SDS-PAGE. All methods show a similar trend, that the highest IgG titers were reached at around day 10-11. There was no significant difference between ELISA and LSPR titers for 13 of the 15 measured samples. Titers estimated using ELISA in samples from day 10 and 11 were higher compared to LSPR. For ELISA, there was a drop in IgG titers after day 11. However, such change was not seen in either LSPR or SDS-PAGE. Interestingly, ELISA gave higher IgG titers than LSPR for day 10 and 11 but the same titers as LSPR after that. The ELISA titers were, therefore, likely overestimated for these two samples. Despite this difference, a high degree of correlation $\left(R^{2}=0.9629\right)$ between these two methods was obtained using linear regression analysis (Figure 8B). The growth curve from LSPR also showed good correlation with SDS-PAGE (sodium dodecyl sulfate-polyacrylamide gel electrophoresis) and a similar correlation coefficient was attained as when comparing LSPR and ELISA. Both ELISA and LSPR use ligand specificity for IgG detection. The small variation in IgG titers between the two methods could be a result of different IgG ligands used for the calibrations. Binding of Human IgG to Anti-human IgG gamma chain and 
monomeric recombinant IgG to Protein A were used for ELISA and LSPR calibration, respectively. Ideally, for both methods, the sample and the standard should contain the same type of IgG.
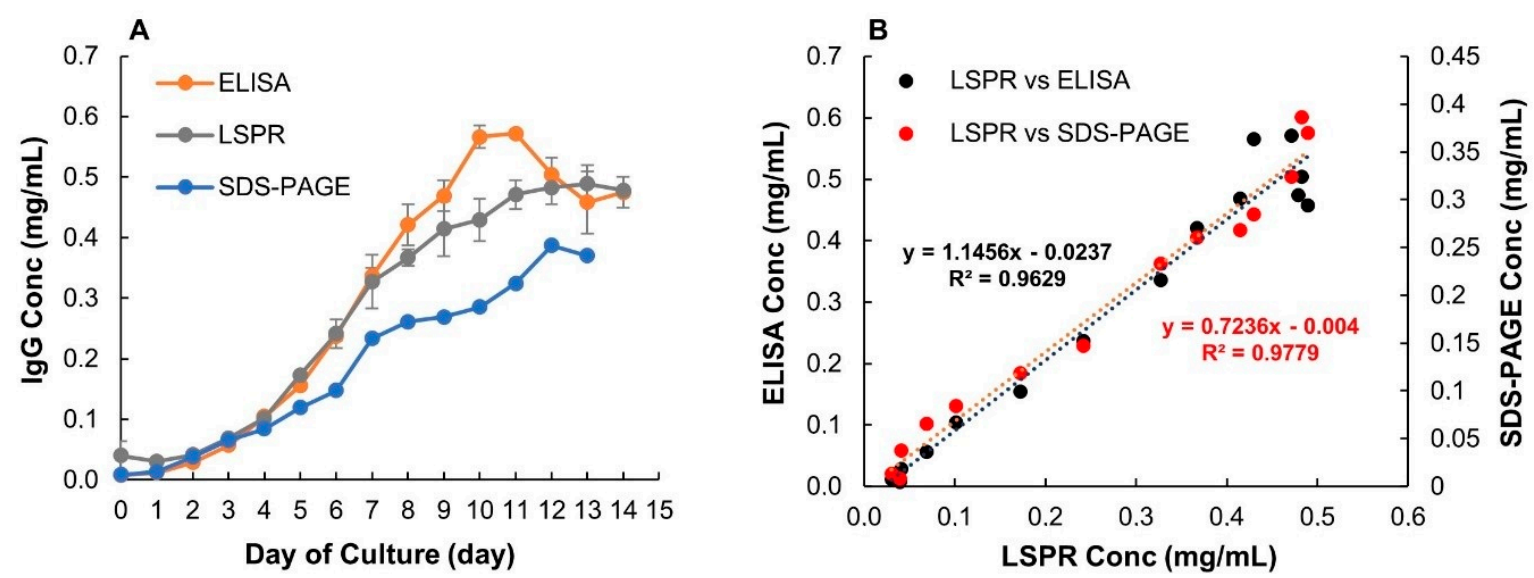

Figure 8. (A) Comparison of IgG titers over cultivation time obtained by ELISA, SDS-PAGE and the developed LSPR sensor. Error bars represent standard deviations ( $\mathrm{n}=3$ and 2 for ELISA and LSPR, respectively. (B) Scatter plot and linear regression analysis for comparison of LSPR versus ELISA and LSPR versus SDS-PAGE.

\section{Conclusions}

In the present work, a reproducible and reliable nanoplasmonic sensor system for rapid monitoring of $\operatorname{IgG}$ titers was evaluated. IgG quantification results from crude cell culture supernatants were highly comparable to conventional protein G-HPLC-UV and ELISA methods. The sample measurement was completed within two minutes by injecting filtrated crude samples directly into the sensor system. The linear dynamic detection range could also be tuned by varying the injection volumes, and samples with concentrations from 0.0015 up to $10 \mathrm{mg} / \mathrm{mL}$ could be quantified. The large dynamic range enabled monitoring of titers commonly seen in both upstream and downstream process steps without any sample dilution. While all the measurements in this work were performed under simulated on-line conditions, the flexible sensor design can enable real-time operation on-line when combined with an auto-sampler that diverts or collects and directs cell-free samples from bioreactors into the flow cell. We thus envision that this novel sensor system can be utilized as a versatile technology for on-line real-time IgG monitoring in both process development and bioproduction.

Author Contributions: Conceptualization, D.A., I.L., C.-F.M. and E.M.; methodology, T.T., O.E., E.M., F.M.; software, E.M.; formal analysis, T.T., E.M., D.A.; investigation, T.T., O.E.; writing-original draft preparation, T.T.; writing-review and editing, D.A., T.T., I.L., C.-F.M., E.M., O.E., R.G., R.S., F.M.; supervision, D.A.; funding acquisition, D.A. All authors have read and agreed to the published version of the manuscript.

Funding: This research was funded by the European Union's Horizon 2020 research and innovation program under the Marie Skłodowska-Curie grant agreement No. 841373 and the Swedish Innovation Agency (VINNOVA), grant numbers 2016-04120 and 2019-00130.

Acknowledgments: Funding from the European Union's Horizon 2020 research and innovation program under the Marie Skłodowska-Curie grant agreement No. 841373 and the Swedish Innovation Agency (VINNOVA), grant numbers 2016-04120 and 2019-00130, are gratefully acknowledged.

Conflicts of Interest: D.A., E.M., I.L. and C.-F.M. are cofounders of ArgusEye AB. 


\section{Appendix A}

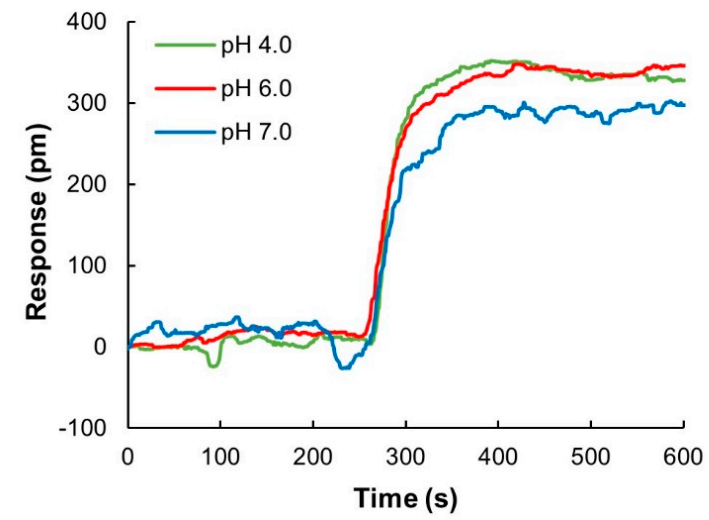

Figure A1. pH scouting for surface functionalization using glass sensor chips that have the same surface chemistry as stainless-steel chips. Protein A was immobilized to the sensor surface using $10 \mathrm{mM}$ sodium acetate $\mathrm{pH}$ 4.0, $10 \mathrm{mM}$ MES $\mathrm{pH} 6.0$ and $10 \mathrm{mM}$ phosphate buffer $\mathrm{pH}$ 7.0.

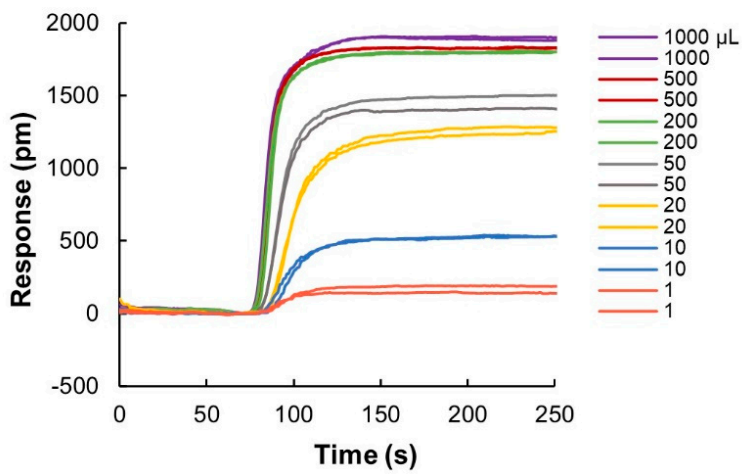

Figure A2. Binding responses of IgG sample $(1 \mathrm{mg} / \mathrm{mL})$ at different injection volumes ranging from $1 \mu \mathrm{L}$ to $1 \mathrm{~mL}$. For injection volumes $>200 \mu \mathrm{L}$, the responses reached maximum binding capacity of the sensor chip. Sensorgrams of two replicates of each injection volumes are depicted with the same color.

\section{References}

1. PAT-A Framework for Innovative Pharmaceutical Development, Manufacturing, and Quality Assurance. 2004. Available online: https://www.fda.gov/media/71012/download (accessed on 29 August 2020).

2. Rathore, A.S.; Bhambure, R.; Ghare, V. Process analytical technology (PAT) for biopharmaceutical products. Anal. Bioanal. Chem. 2010, 398, 137-154. [CrossRef]

3. Glassey, J.; Gernaey, K.V.; Clemens, C.; Schulz, T.W.; Oliveira, R.; Striedner, G.; Mandenius, C.F. Process analytical technology (PAT) for biopharmaceuticals. Biotechnol. J. 2011, 6, 369-377. [CrossRef]

4. Guerra, A.; Von Stosch, M.; Glassey, J. Toward biotherapeutic product real-time quality monitoring. Crit. Rev. Biotechnol. 2019, 39, 289-305. [CrossRef]

5. Kesik-Brodacka, M. Progress in biopharmaceutical development. Biotechnol. Appl. Biochem. 2018, 65, 306-322. [CrossRef]

6. Tsumoto, K.; Isozaki, Y.; Yagami, H.; Tomita, M. Future perspectives of therapeutic monoclonal antibodies. Immunotherapy 2019, 11, 119-127. [CrossRef] [PubMed]

7. Shukla, A.A.; Wolfe, L.S.; Mostafa, S.S.; Norman, C. Evolving trends in mAb production processes. Bioeng. Transl. Med. 2017, 2, 58-69. [CrossRef] [PubMed]

8. Kunert, R.; Reinhart, D. Advances in recombinant antibody manufacturing. Appl. Microbiol. Biotechnol. 2016, 100, 3451-3461. [CrossRef] [PubMed]

9. Huang, Y.-M.; Hu, W.; Rustandi, E.; Chang, K.; Yusuf-Makagiansar, H.; Ryll, T. Maximizing productivity of $\mathrm{CHO}$ cell-based fed-batch culture using chemically defined media conditions and typical manufacturing equipment. Biotechnol. Prog. 2010, 26, 1400-1410. [CrossRef] [PubMed] 
10. Reinhart, D.; Damjanovic, L.; Kaisermayer, C.; Kunert, R. Benchmarking of commercially available CHO cell culture media for antibody production. Appl. Microbiol. Biotechnol. 2015, 99, 4645-4657. [CrossRef]

11. Mushens, R.E.; Guest, A.R.; Scott, M.L. Quantitation of monoclonal antibodies by ELISA: The use of purified mouse IgG and mouse IgM monoclonal antibodies as standards in a quantitative ELISA measuring monoclonal antibodies produced by cell culture. J. Immunol. Methods 1993, 162, 77-83. [CrossRef]

12. Horak, J.; Ronacher, A.; Lindner, W. Quantification of immunoglobulin G and characterization of process related impurities using coupled Protein A and size exclusion high performance liquid chromatography. J. Chromatogr. A 2010, 1217, 5092-5102. [CrossRef] [PubMed]

13. Fernández, L.P.; Calvo, L.; Viña, L. Development and Validation of an Affinity Chromatography-Protein G Method for IgG Quantification. Int. Sch. Res. Not. 2014, 2014, 48710. [CrossRef]

14. Frostell, Å.; Mattsson, A.; Eriksson, Å.; Wallby, E.; Kärnhall, J.; Illarionova, N.B.; Nilsson, C.E. Nine surface plasmon resonance assays for specific protein quantitation during cell culture and process development. Anal. Biochem. 2015, 477, 1-9. [CrossRef]

15. Zschätzsch, M.; Ritter, P.; Henseleit, A.; Wiehler, K.; Malik, S.; Bley, T.; Walther, T.; Boschke, E. Monitoring bioactive and total antibody concentrations for continuous process control by surface plasmon resonance spectroscopy. Eng. Life Sci. 2019, 19, 681-690. [CrossRef]

16. Yu, Y.; Mitchell, S.; Lynaugh, H.; Brown, M.; Nobrega, R.P.; Zhi, X.; Sun, T.; Caffry, I.; Cao, Y.; Yang, R.; et al. Understanding ForteBio's Sensors for High-Throughput Kinetic and Epitope Screening for Purified Antibodies and Yeast Culture Supernatant. J. Biomol. Screen. 2016, 21, 88-95. [CrossRef]

17. Wang, Y.; Feng, P.; Sosic, Z.; Zang, L. Monitoring Glycosylation Profile and Protein Titer in Cell Culture Samples Using ZipChip CE-MS. J. Anal. Bioanal. Technol. 2017, 8. [CrossRef]

18. Pedersen, M.E.; Østergaard, J.; Jensen, H. In-Solution IgG Titer Determination in Fermentation Broth Using Affibodies and Flow-Induced Dispersion Analysis. ACS Omega 2020, 5, 10519-10524. [CrossRef] [PubMed]

19. Swartz, A.R.; Chen, W. Rapid Quantification of Monoclonal Antibody Titer in Cell Culture Harvests by Antibody-Induced Z-ELP-E2 Nanoparticle Cross-Linking. Anal. Chem. 2018, 90, 14447-14452. [CrossRef] [PubMed]

20. Chemmalil, L.; Prabhakar, T.; Kuang, J.; West, J.; Tan, Z.; Ehamparanathan, V.; Song, Y.; Xu, J.; Ding, J.; Li, Z. Online/at-line measurement, analysis and control of product titer and critical product quality attributes (CQAs) during process development. Biotechnol. Bioeng. 2020. [CrossRef]

21. Willets, K.A.; Van Duyne, R.P. Localized Surface Plasmon Resonance Spectroscopy and Sensing. Annu. Rev. Phys. Chem. 2007, 58, 267-297. [CrossRef]

22. Bhagawati, M.; You, C.; Piehler, J. Quantitative Real-Time Imaging of Protein-Protein Interactions by LSPR Detection with Micropatterned Gold Nanoparticles. Anal. Chem. 2013, 85, 9564-9571. [CrossRef] [PubMed]

23. Liu, Y.; Zhang, N.; Li, P.; Yu, L.; Chen, S.; Zhang, Y.; Jing, Z.; Peng, W. Low-Cost Localized Surface Plasmon Resonance Biosensing Platform with a Response Enhancement for Protein Detection. Nanomaterials 2019, 9, 1019. [CrossRef] [PubMed]

24. Chavane, N.; Jacquemart, R.; Hoemann, C.D.; Jolicoeur, M.; De Crescenzo, G. At-line quantification of bioactive antibody in bioreactor by surface plasmon resonance using epitope detection. Anal. Biochem. 2008, 378, 158-165. [CrossRef] [PubMed]

25. Hampson, G.; Ward, T.H.; Cummings, J.; Bayne, M.; Tutt, A.; Cragg, M.S.; Dive, C.; Illidge, T.M. Validation of an ELISA for the determination of rituximab pharmacokinetics in clinical trials subjects. J. Immunol. Methods 2010, 360, 30-38. [CrossRef] [PubMed]

26. Liu, X.F.; Wang, X.; Weaver, R.J.; Calliste, L.; Xia, C.; He, Y.J.; Chen, L. Validation of a gyrolab ${ }^{\mathrm{TM}}$ assay for quantification of rituximab in human serum. J. Pharmacol. Toxicol. Methods 2012, 65, 107-114. [CrossRef]

27. Satzer, P.; Jungbauer, A. High-capacity protein A affinity chromatography for the fast quantification of antibodies: Two-wavelength detection expands linear range. J. Sep. Sci. 2018, 41, 1791-1797. [CrossRef]

Publisher's Note: MDPI stays neutral with regard to jurisdictional claims in published maps and institutional affiliations. 